\title{
Polyethylene Glycol/Carbon Black Shape-Stable Phase Change Composites for Peak Load Regulating of Electric Power System and Corresponding Thermal Energy Storage
}

\author{
Xiang Lu, ${ }^{1 *}$ Huanyu Liu,,${ }^{2}$ Vignesh Murugadoss, ${ }^{4}$ Ilwoo Seok, ${ }^{5}$ Jintao Huang, ${ }^{3}$ Jong E. Ryu ${ }^{6}$ and Zhanhu Guo ${ }^{4 *}$
}

In this work, a unique electrically conductive polyethylene glycol (PEG)/carbon black (CB) shape-stable phase change composite (SSPCC) for peak load regulating of electric power system was prepared via a vacuum impregnation approach. Scanning electron microscope (SEM), differential scanning calorimeter (DSC) and X-ray diffraction (XRD) were used to study the micro morphology, crystallization behavior, crystallization structure and thermal properties. Leakage test and DSC results showed that when the PEG content in PEG/CB SSPCC was as high as $86 \mathrm{wt} \%$, the phase change enthalpy and relative enthalpy efficiency were up to $147.7 \mathrm{~J} / \mathrm{g}$ and $95.1 \%$, respectively. Fourier transform infrared (FTIR) and XRD showed no chemical reaction between CB and PEG. Thermal cycling test showed that the PEG/CB SSPCC had excellent thermal stability and thermal reliability. Moreover, the good electro-tothermal conversion ability of PEG/CB SSPCC $(0.2 \mathrm{~S} / \mathrm{m})$ provided rich possibilities for peak load regulating of electric power system and corresponding thermal energy storage.

Keywords: Carbon black; Polyethylene glycol; Electro-to-thermal; Thermal energy storage; SSPCC

Received 24 October 2019, Accepted 6 January 2020

DOI: $10.30919 / \mathrm{es} 8 \mathrm{~d} 901$

\section{Introduction}

With the expansion of economic society and the increase of population, the demand for energy around the world is increasing day by day ${ }^{1-3}$ And the limited fossil energy in nature is gradually decreasing due to the massive consumption of humans in the last hundred years. ${ }^{4,5}$ Renewable sources on the earth, for example, solar power, water power, etc., have raised extensive attention by researchers due to their high renewal rate and sustainablity in nature. In many cases, energy supply and demand are unbalanced and have a strong time dependence. Thus, in addition to developing and utilizing new renewable sources, improving the use efficiency of existing energy (such as fossil energy and renewable energy) is also an important issue. ${ }^{6-9}$
As one of the most important energy forms in modern society, electric energy, generated by hydroelectric power, thermal power, and wind power, etc., is widely used in all aspects of national life, and it also provides great convenience for human life. For a variety of reasons, the generation of electric energy is usually huge and continuous. But the human demands for electric energy have a strong time dependence. Generally speaking, the daily life and work of human beings is mainly carried out from 8 am to $10 \mathrm{pm}$, therefore, the demand for electric energy during this period is very high. But from $10 \mathrm{pm}$ to $8 \mathrm{am}$ at the following day, most people and machines are at rest, and the demand for electric energy is relatively low. At night, the generated electric energy is largely idle. Thus, it is necessary to store the idle electric energy. In

\footnotetext{
${ }^{1}$ Key Laboratory of Polymer Processing Engineering of the Ministry of Education, National Engineering Research Center of Novel Equipment for Polymer Processing, Guangdong Key Laboratory of Technique and Equipment for Macromolecular Advanced Manufacturing, South China University of Technology, Guangzhou, 510641, China

${ }^{2}$ School of Mechanical Engineering, Dongguan University of Technology, Dongguan 523808, China

${ }^{3}$ Department of Materials Science and Engineering, Southern University of Science and Technology, Shenzhen, Guangdong 518055, China

${ }^{4}$ Integrated Composites Laboratory (ICL), Department of Chemical \& Biomolecular Engineering, University of Tennessee, Knoxville, USA

${ }^{5}$ Mechanical Engineering, Arkansas State University, Jonesboro, Arkansas, 72401, USA

${ }^{6}$ Department of Mechanical and Aerospace Engineering, North Carolina State University, Raleigh, NC 27695, USA

*E-mail: luxiang_1028@163.com(X.Lu); zguo10@utk.edu(Z.Guo)
} 
order to ease the situation, the electric energy storage technology based on batteries, ${ }^{10}$ supercapacitors, ${ }^{11}$ etc. has emerged.

Thermal energy, as the biggest energy source in nature, plays a very important role in human daily life. Consequently, to efficiently use the relatively surplus and cheap electric energy at night and convert it into the heat that can be used directly in the human daily life is a meaningful alternative way. In addition, compared to electrical energy and chemical energy storage technologies, thermal energy storage (TES) has a longer history, and it is simpler and easier to comply. ${ }^{12-15}$ Furthermore, the use of phase change materials (PCMs) based on latent heat storage technology has raised extensive attention recently owing to its comprehensive advantages of constant temperature, easy process control, high energy storage density (about 15 times of sensible heat storage materials), and repeated use. ${ }^{16-18} \mathrm{Up}$ to now, thermal energy storage technology based PCMs has become the most important and common way to store heat energy in the area of waste-heat recovery in industry and solar energy, etc..

Among thousands of PCMs that have been discovered, organic PCM, such as polyethylene glycol (PEG), ${ }^{19,20}$ fatty acid, ${ }^{21}$ capric-stearic acid ${ }^{22}$ and paraffin, ${ }^{23}$ etc., has become a research hotspot due to its low cost, high phase transition enthalpy, moderate phase transition temperature, little supercooling, etc. However, the biggest drawback for most organic PCMs is the typical solid-liquid transition and leakage risk during the energy storage and release. Therefore, a variety of strategies, including chemical crosslinking, polymer shaping, microcapsule coating and porous materials adsorbing etc., have been applied to fabricate shape-stable organic PCMs, which can maintain their original shape and avoid the risk of leakage during use. For example, Sundararajan et al. ${ }^{24}$ synthesized a series of PEG-based hyperbranched polyurethanes as shape-stable PCMs (SSPCCs) using oligomeric $\mathrm{A}_{2}+\mathrm{B}_{3}$ approach for thermal energy storage. Guo et al. ${ }^{25}$ prepared diatomite stabilized paraffin/wood flour/highdensity polyethylene PCM with acceptable TES performance and good mechanical strength in building energy conversion. Jiang et al. ${ }^{26}$ prepare new paraffin wax/poly (methyl methacrylate-co-methyl acrylate)/nano $\quad \mathrm{Al}_{2} \mathrm{O}_{3}$ microencapsulated PCMs through the emulsion polymerization, and discussed their potential applications in energy-saving building. In addition to this, porous materials have also widely been used to prepare SSPCCs via a vacuum impregnation approach to improve the shape stability, and this approach was easy to operate and industrialize. For example, Tang et al. ${ }^{27}$ prepared PEG/graphene oxide aerogel (GOA) SSPCCs by introducing PEG into GAs via vacuum impregnation, and they pointed out that the prepared PCM exhibited good photo-to-thermal energy conversion property. Yuan et al. ${ }^{28}$ prepared a erythritol/expanded graphite (EG)
SSPCC via three-step technique for mid-temperature thermal energy storage.

As described above, the modified organic SSPCCs can efficiently store the thermal energy because of their high phase transition enthalpy, moderate phase transition temperature and little super-cooling. According to the Joule's Law, when the current passes through the conductor, heat is generated, and the generated heat $(\mathrm{Q})$ can be calculated by $\operatorname{Eqn}(1)$ :

$$
\mathrm{Q}=I^{2} R t
$$

where $I$ is the current intensity in the circuit, $R$ is the resistance value of the conductor, and $t$ is the time. Based on this, if the prepared organic SSPCCs are electrically conductive, it may be used for electro-to-thermal energy conversion to achieve peak load regulating of electric power system at night to improve the use efficiency of idle electric energy.

Compared with carbon nanotubes and graphene, conductive carbon black (CB) is an inexpensive and relatively efficient electrically conductive filler, has the characteristics of small particle size (nanoscale), large specific surface area and rough surface, and is widely used in electrically conductive polymer composites. ${ }^{29-31}$ Theoretically, there will be strong interactions and adsorptions between $\mathrm{CB}$ nanoparticles and organic PCM molecular chains because of the small particle size (nanoscale), large specific surface area and rough surface of $\mathrm{CB}$. The organic PCM/CB composites with high electrical conductivity can be obtained via the simple vacuum impregnation, and the obtained SSPCCs may be applied in electric-to-thermal energy conversion and storage according to the Joule's Law. PEG, as a typical solid-liquid transition organic PCM, has received extensive attention from researchers recently. However, as far as we know, the application of CB to the field of electrical conductivity PEGbased PCMs via electro-to-thermal conversion for peak load regulating of electric power system has been rarely reported.

In this study, PEG/CB composites were prepared via the simple vacuum impregnation and the effects of $\mathrm{CB}$ on the shape-stable performance, thermal properties, crystallization behavior, electrical conductivity, and electro-to-thermal energy conversion behavior of the obtained PEG/CB SSPCC were studied. The research results provide rich theoretical support for expanding the application of PCMs in the aspect of peak load regulating of electric power system, which has important scientific and practical significances.

\section{Experiment}

\subsection{Materials}

Polyethylene glyol (PEG-10000) was provided by Shanghai Aladin Industrial Co., Ltd.. Conductive carbon black (ENSACO 250G: Brunauer-Emmett-Teller (Bet) surface $=65$ $\mathrm{m}^{2} / \mathrm{g}$ ) was purchased from TIMICAL Co., Ltd., Switzerland.

\subsection{Fabricating Method of PEG@CB SSPCC}


The PEG/CB SSPCC was fabricated by a direct vacuum impregnation method. Briefly, $3 \mathrm{~g}$ CB powder and $22 \mathrm{~g}$ solid PEG were put into a glass container first, and then moved in a vacuum oven to achieve the adsorption saturation at $80^{\circ} \mathrm{C}$ for $12 \mathrm{~h}$ under high vacuum. After the vacuum adsorption process, the specimen was placed onto a sheet of filter paper, and then put into a vacuum oven to remove PEG residue at the specimen surface at $80^{\circ} \mathrm{C}$ for $2 \mathrm{~h}$. The filter paper changed periodically and ended when no PEG leaked from the sample. Finally, PEG/ CB SSPCC was obtained and identified as PEG@CB. The PEG loading (w) in the PEG@CB was calculated by Eqn. (2):

$$
\mathrm{w}=\frac{m_{2}-m_{1}}{m_{2}} \times 100 \%
$$

where $m_{1}$ is the quantity of pristine $\mathrm{CB}$ and $m_{2}$ is the quantity of the prepared PEG@CB composite.

\subsection{Characterization Methods}

Fourier transform infrared (FT-IR) test was carried out by an equipment from PerkinElmer labeled by Spectrum 2000 over the scanning range $4000-400 \mathrm{~cm}^{-1}$. The BET method was introduced to evaluate the pore volume and specific surface area, and the samples were tested on BELSORP-max (Microtrac BEL, Japan) at $77 \mathrm{~K}$. Morphology observations of $\mathrm{CB}$ and PEG@CB were performed on a field emission scanning electron microscopy (Quanta FEG 250). XRD tests (diffraction angle $5-60^{\circ}$ ) were conducted on a Bruker D8 Advance powder diffractor meter. The heat storage behavior of samples was characterized by a DSC equipment (Netzsch 204c). The samples were heated and cooled under a nitrogen atmosphere in test at a rate of $10{ }^{\circ} \mathrm{C} / \mathrm{min}$. TGA was performed to evaluate the thermal stability by Netzsch TG209. The samples were tested from 30 to $600{ }^{\circ} \mathrm{C}$ and heated under nitrogen flow at a rate of $10{ }^{\circ} \mathrm{C} / \mathrm{min}$. The accelerated thermal cycling test was used to evaluate the thermal reliability and reusability of PEG@CB via high-low temperature chamber. The volume electrical resistivity was measured at $25^{\circ} \mathrm{C}$ using an electrical conductivity analyzer with four probes (RTS-4). The thermal conductivity of pure PEG and PEG@CB were measured at $25^{\circ} \mathrm{C}$ by the transient plane source method using a laser thermal conductivity analyzer (LFA-427).The electro- thermal conversion and storage behaviors of the obtained PEG@CB were studied via a self-built circuit. Infrared thermography camera was used for the collection of temperature change during test.

\section{Results and Discussion}

\subsection{Leakage Test}

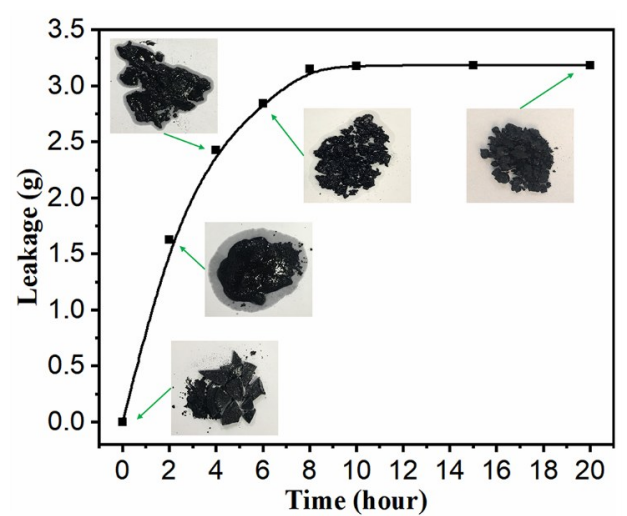

Fig. 1 The quantity of PEG leakage at different time periods.

As described in Section 2.2, $3 \mathrm{~g} \mathrm{CB}$ powder and $22 \mathrm{~g}$ solid PEG were put into a glass container first, and then moved in a vacuum oven to achieve the adsorption saturation at $80^{\circ} \mathrm{C}$ for $12 \mathrm{~h}$ under high vacuum. For calculating the loading of $\mathrm{PEG}$ in PEG@CB without any leakage, leakage tests were carried out on the obtained PEG/CB composite. The specimen was placed onto a sheet of filter paper. The quantity of leaked PEG at different time ranging from 0 to 20 hours is shown in Fig. 1. Also, the corresponding digital photos of specimen during leakage are presented. At the beginning, the specimen temperature is near the room temperature, and the sample is in a solid state. After being heated, the un-adsorbed PEG in PEG/ $\mathrm{CB}$ composite was quickly melted to a liquid state, and was adsorbed by the filter paper on the bottom. As shown in Fig. 1, the quantity of the leaked PEG increased with increasing the test time. At about 8 hours, the leaked PEG quantity maintained at around $3.2 \mathrm{~g}$ and the leaking quantity reached its equilibrium state. This indicates that no more PEG leaks out from the PEG/ $\mathrm{CB}$ composite further. The phenomenon shows that un-leaked

Table 1 Comparison of Maximum PEG ratio in PCM in literatures.

\begin{tabular}{cccc}
\hline PCM & Method & Maximum ratio & Ref. \\
\hline PEG/Diatomite & Vacuum impregnation & $50 \%$ & 32 \\
PEG/Diatomite pretreatment & Direct impregnation & $55 \%$ & 33 \\
PEG/Diatomite pretreatment & Vacuum impregnation & $70 \%$ & 34 \\
PEG/ Biological porous carbon & Vacuum impregnation & $85.36 \%$ & 1 \\
PEG/Expanded perlite & Vacuum impregnation & $73.93 \%$ & 35 \\
PEG/AC & Vacuum impregnation & $80 \%$ & 36 \\
PEG/EG & Vacuum impregnation & $87 \%$ & 37 \\
PEG@CB & Vacuum impregnation & $86 \%$ & Present work \\
\hline
\end{tabular}



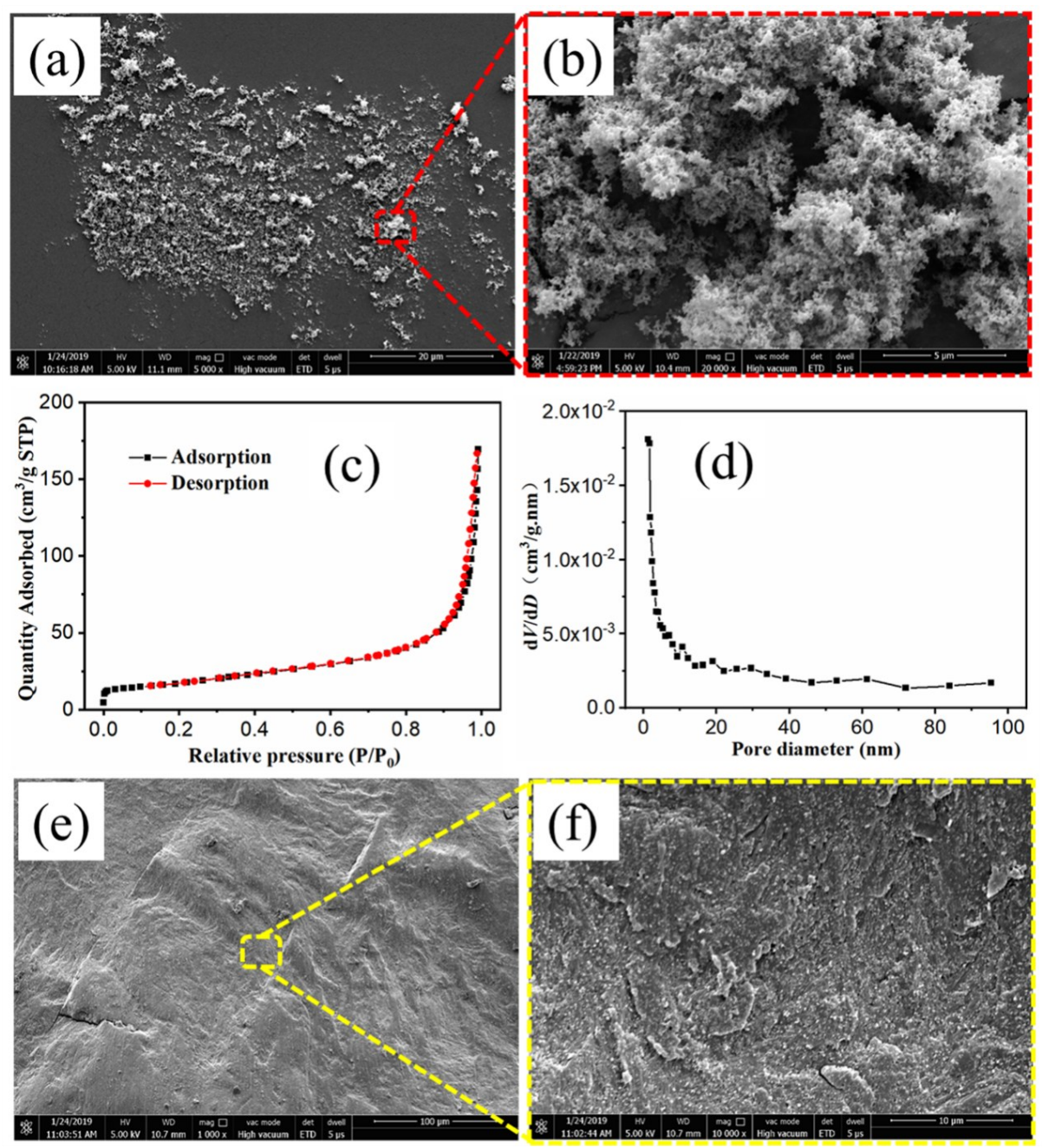

Fig. 2 (a-b) SEM pictures of $\mathrm{CB}$, (c) nitrogen adsorption/desorption isotherm curves for $\mathrm{CB}$, (d) the pore diameter distribution of $\mathrm{CB}$, and (e-f) SEM images of PEG@CB.

PEG was well adsorbed by CB. According to Eqn. (2), the loading of PEG in the PEG@CB is up to $86 \mathrm{wt} \%$, and it is higher than most of the values in the previously reported work, refer to Table 1.

\subsection{Morphology Observations}

The above leakage test reveals that PEG@CB containing only $14 \mathrm{wt} \% \mathrm{CB}$ as shape supporting material owns excellent shape stability. This high adsorption capacity of CB on PEG must be directly related to the microstructure of $\mathrm{CB}$. Therefore, it is necessary to study the microscopic morphology of $\mathrm{CB}$ and PEG@CB. Figs. 2(a-b) presents the SEM images of CB, Fig. 2(c) shows the nitrogen adsorption/desorption isotherm curve and Fig. 2(d) shows the pore diameter distribution of CB. It can be seen that the CB is a fluffy powder. Due to the surface tension, hydrogen bond as well as capillary force, PEG will be absorbed into the surface in homogeneity and pores of $\mathrm{CB}$. Figs. 2(c-d) reveals that $C B$ has a pore diameter of 1-100 nm. The average value of diameter is approximate $15.7 \mathrm{~nm}$ and specific surface area is near $63.2 \mathrm{~m}^{2} / \mathrm{g}$. The typical mesoporous structure of CB is beneficial to absorb melting PEG segment. The SEM images of PEG@CB are shown in Figs. 2(e\&f), a nonporous PEG@CB is observed. It indicates that the melted PEG is easily absorbed by CB due to its porous capillary force and vacuum negative pressure during the vacuum impregnation process.

\subsection{FTIR}

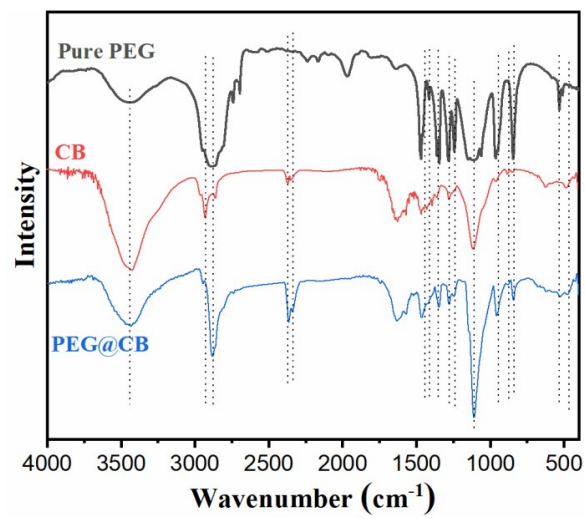

Fig. 3 FITR spectra of PEG@CB, PEG and CB. 
Fig. 3 shows the FI-TR spectrum of PEG@CB, PEG and CB. From FI-TR spectra of pure PEG, the characteristic absorption peaks at wave number of $3440 \mathrm{~cm}^{-1}$ and 1107 $\mathrm{cm}^{-1}$ belong to $-\mathrm{O}-\mathrm{H}$ and $-\mathrm{C}-\mathrm{O}$ stretching vibrations, respectively. ${ }^{38,39}$ Additionally, the peaks at a wave number of $2887 \mathrm{~cm}^{-1}, 1469 \mathrm{~cm}^{-1}, 1342 \mathrm{~cm}^{-1}, 961 \mathrm{~cm}^{-1}, 842 \mathrm{~cm}^{-1}$ are due to the $\mathrm{C}-\mathrm{H}$ vibrations. ${ }^{39}$ For $\mathrm{CB}$, the observed strong characteristic absorption peaks at around $3440 \mathrm{~cm}^{-1}$ and $1640 \mathrm{~cm}^{-1}$ belong to the $\mathrm{O}-\mathrm{H}$ stretching vibration and bending vibration, respectively. ${ }^{40}$ This indicates a lot of hydrophilic groups on the surface of the $\mathrm{CB}$ particles, which make it possible for $\mathrm{CB}$ and $\mathrm{PEG}$ to form a strong hydrogen bond interaction. Compared with PEG and $\mathrm{CB}$, FT-IR curve of PEG@CB is a simple superposition of pure PEG and CB curve. In PEG@CB spectra, no new characteristic peaks are found and there is no significant peak shift. It implies that no chemical reaction between PEG and CB and they are only physically combined with each other.

\subsection{XRD}

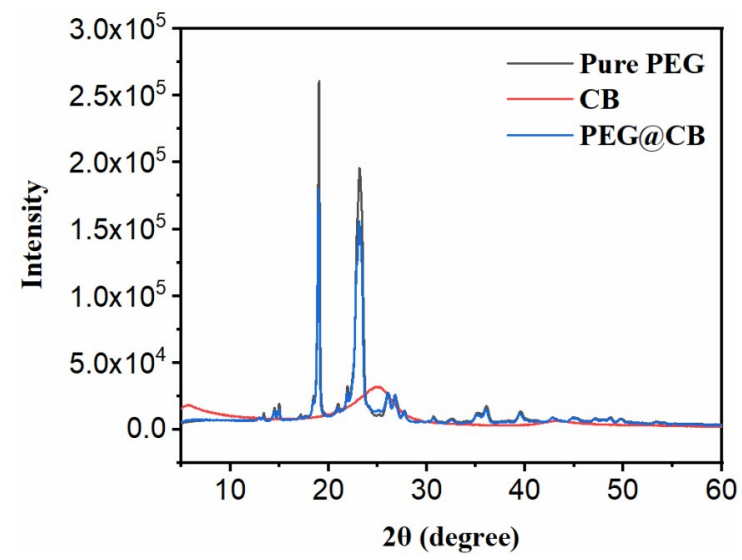

Fig. 4 XRD patterns of pure PEG, CB, PEG@CB.

Crystallization property is an important performance of PCMs. The crystal structures of pure PEG, CB and PEG@CB were characterized by XRD. The XRD patterns of pure PEG, PEG@CB and CB are present in Fig. 4. For pure PEG, two sharp peaks are found, one peak at $19.1^{\circ}$ (120) and the other one peak at $23.1^{\circ}$ (132). It implies that pure PEG has good crystallization. However, CB pattern shows a weak and flat diffraction peak at approximately $25.0^{\circ}$, which reveals poor crystallization capability of $\mathrm{CB}$. PEG@CB pattern has similar peaks to pure PEG, and there is no newpeak other than characteristic peaks of $\mathrm{CB}$ and PEG. The above results indicate that the addition of $\mathrm{CB}$ does not significantly affect the crystalline regions of PEG chains in PEG@CB. Both XRD and FITR results confirm that $\mathrm{PEG}$ and $\mathrm{CB}$ have no chemical reaction, and they are physically combined with each other. Hence, CB and PEG can well preserve their respective structural integrity.

\subsection{Phase Change Properties}

In this study, phase change heat storage behaviors of PEG@CB and pure PEG were characterized by DSC. Fig. 5 displays the corresponding curves between 0 and $100{ }^{\circ} \mathrm{C}$. The corresponding melting latent heat $\left(\Delta H_{m}\right)$, melting temperature $\left(\mathrm{T}_{m}\right)$, freezing latent heat $\left(\Delta H_{f}\right)$ and freezing temperature $\left(\mathrm{T}_{f}\right)$ are shown in Table 1. For the purpose of studying the effect of $\mathrm{CB}$ on phase change behavior of PEG, the theoretical latent heat melting enthalpy $\left(\Delta H_{t m}\right)$, theoretical latent heat freezing enthalpy $\left(\Delta H_{t f}\right)$, and relative enthalpy efficiency $(\lambda)$ of pure PEG and PEG@CB (displayed in Table 1) are calculated from curves in Fig. 5. The $\Delta H_{t m}, \Delta H_{t f}$ and $\lambda$ are calculated as follows:

$$
\begin{aligned}
\Delta H_{t m} & =w \cdot \Delta H_{m(\text { Pure } P E G)} \\
\Delta H_{t f} & =w \cdot \Delta H_{f(\text { Pure PEG })} \\
\lambda & =\frac{\Delta H_{m}}{\Delta H_{t m}}
\end{aligned}
$$

where $\Delta H_{m}$ is the measured melting enthalpy from DSC, and $w$ is the weight fraction of PEG in the PEG@CB.

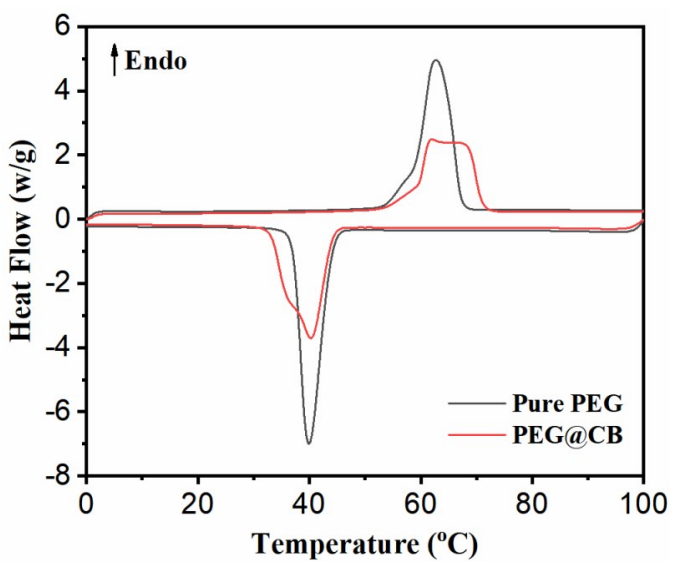

Fig. 5 DSC curves of pure PEG and PEG@CB.

As presented in Table 2 and Fig. 5, $\mathrm{T}_{m}$ of PEG in PEG@CB decreases to $62.7^{\circ} \mathrm{C}$, and $\mathrm{T}_{f}$ of $\mathrm{PEG}$ in PEG@CB is not remarkably influenced with the addition of CB. At the same time, $\Delta H_{m}$ and $\Delta H_{f}$ of PEG@CB decrease to 147.7 and $146.0 \mathrm{~J} / \mathrm{g}$, respectively. Actually, $\Delta H_{t m}$ and $\Delta H_{t f}$ should be 155.4 and $148.4 \mathrm{~J} / \mathrm{g}$, respectively. Why are all the $\Delta H_{m}$ and $\Delta H_{f}$ lower than the $\Delta H_{t m}$ and $\Delta H_{t t}$, respectively? The reason may be as follows: the crystallization of polymer from the molten state can be divided into two stages, crystal nucleation and crystal growth, the CB not only acts as a nucleating agent to enhance the crystallization of PEG segments, but also limits the movement of PEG segments due to the interaction between $\mathrm{CB}$ and $\mathrm{PEG}$ segments, thereby reduces the crystallinity of PEG component in PEG@CB. In this system, the $\Delta H_{m}$ and $\Delta H_{f}$ of 
PEG@CB are both lower than its $\Delta H_{t m}$ and $\Delta H_{t}$, it can be attributed to the fact that $\mathrm{CB}$ hinders the movement of $\mathrm{PEG}$ segments more dominant than the nucleation promoting effect of PEG segments. Even so, the $\lambda$ value of PEG@CB reaches up to $95.1 \%$, which is bigger than most of the previously reported $\lambda$ values. ${ }^{41,42}$ All the above results indicate that $\mathrm{CB}$ is a highly efficient support material for preparing PEG-based SSPCCs.

\subsection{Thermal Reliability and Reusability}

The good thermal reliability, reusability and thermal stability are paramount parameters for long-term use of SSPCCs. Thus, thermal cycling process was accelerated by using high-low temperature chamber and thermogravimetric analysis, for simulating long term use of PEG@CB. Figs. 6 (a, b\&c) shows the FT-IR, DSC, XRD curves of PEG@CB respectively. The TG curves of pure PEG and PEG@CB are displayed in Fig. 6 (d). The changes of $\Delta H_{m}$ and $\Delta H_{f}$ after thermal cycling process were evaluated by the relative coefficient $\eta_{m}$ (melting) and $\eta_{f}$ (freezing) value, respectively. Smaller values of $\eta_{m}$ and $\eta_{f}$ mean a better reusability. And the corresponding thermal parameters are listed in Table 3. As displayed in Figs. 6(a\&c), for PEG@CB, the characteristic band positions remain the same after 100 thermal cycles. It implies that the prepared SSPCC is of excellent structural stability. DSC curves of PEG@CB are almost overlapped before and after 100 thermal cycles, as shown in Fig. 6(b), which indicates that the obtained PEG@CB presents good thermal reliability. At the same time, as listed in Table 2, the $\Delta H_{m}$ and $\Delta H_{f}$ of PEG@CB do not change after 100 thermal cycles. In addition, the $\eta_{m}$ and $\eta_{f}$ values of PEG@CB are $2.2 \%$ and $-0.4 \%$, respectively, for melting process and freezing process after 100 thermal cycles. The slight shift of $\Delta H_{m}$ and $\Delta H_{f}$ can be ignored for thermal energy storage applications during the long time use. TGA and the corresponding DTG curves for PEG@CB are shown in Fig. 6 (d), and Table 4 shows the maximum degradation temperature $\left(\mathrm{T}_{\max }\right)$, the weight loss at various temperatures, and the onset degradation temperature $\left(\mathrm{T}_{5}\right)$, respectively. $\mathrm{T}_{5}$ is assigned to 5 wt $\%$ degradation, and $\mathrm{T}_{\max }$ is the peak temperature of the DTG curve. $\mathrm{T}_{5}$ of PEG@CB increases to $382.5{ }^{\circ} \mathrm{C}$ while pure PEG is $380.7^{\circ} \mathrm{C}$. But the $\mathrm{T}_{\text {max }}$ decreases from $421.6{ }^{\circ} \mathrm{C}$ (pure PEG) to $413.5^{\circ} \mathrm{C}$ (PEG@CB). This is because the introduction of $\mathrm{CB}$ enhanced the thermal conductivity. Moreover, the shift of mass loss below $100{ }^{\circ} \mathrm{C}$ for all samples is not obvious. On the whole, for PEG@CB, no degradation occurs in the phase change temperature range. It shows that the PEG@CB presents excellent thermal stability in the application temperature range. At last, all the test results illustrate that the obtained PEG@CB SSPCC has excellent thermal reliability and reusability for a long term use.

\subsection{Electro-Thermal Energy Conversion and Storage}

For the PEG@CB, in order to achieve the high-speed thermal energy storage via electro-to-thermal conversion process, the thermal conductivity and electrical conductivity of PEG@CB are significant parameter. The thermal conductivity and volume electrical conductivity of samples are displayed in Figs. 7 (a\&b), respectively. The thermal conductivity of pure PEG is about $0.25 \mathrm{~W} / \mathrm{mK}$, and the volume electrical conductivity of pure PEG is as low as $3 \times 10^{-11} \mathrm{~S} / \mathrm{m}$, it is a typical insulator. Interestingly, with the introduction of $\mathrm{CB}$, the thermal conduvtivity of PEG@CB increases to $1.32 \mathrm{~W} / \mathrm{mK}$, and the volume electrical conductivity of compacted PEG@CB reaches $0.2 \mathrm{~S} / \mathrm{m}$, it shows that the compacted PEG@CB is a good conductor. The good thermal conductivity and electrical conductivity of PEG@CB can provide rich possibilities for the electro-to-thermal conversion and storage.

For the purpose of observing the thermal energy storage behavior of the electrical conductive PEG@CB SSPCC during the electro-to-thermal conversion, we built an electro-to-thermal conversion experimental device, and the temperature change of PEG@CB was recorded by the infrared thermography camera. Fig. 8(a) and Fig. 8(b) show the digital photo of self-built electro-to-thermal conversion experimental device and the simulation diagram for electroto-thermal conversion and storage, respectively. The temperature curves as a function of time for PEG@CB during the test are shown in Fig. 9. The real-time temperature was documented by the infrared thermography camera. The corresponding infrared thermographic images of PEG@CB are shown in Fig. 10, the red portion refers to the high temperature while the blue portion means the low temperature. Initially, no heat was generated in the circuit due to the turned off power, and the sample temperature was the same as the ambient temperature $\left(25.3{ }^{\circ} \mathrm{C}\right)$. Thus, the image is mainly blue (as shown in Fig. 10a).

After turning on the power, according to the Joule's Law, a large amount of heat was generated and stored by the

Table 2 Phase change performances of PEG@CB and pure PEG.

\begin{tabular}{crrccc}
\hline Specimen & $\mathrm{T}_{m}\left({ }^{\circ} \mathrm{C}\right)$ & $\Delta H_{m}(\mathrm{~J} / \mathrm{g})$ & $\mathrm{T}_{f}\left({ }^{\circ} \mathrm{C}\right)$ & $\Delta H_{f}(\mathrm{~J} / \mathrm{g})$ & $\lambda(\%)$ \\
\hline Pure PEG & 64.0 & 180.7 & 41.9 & 172.5 & - \\
PEG@CB & 62.7 & 147.7 & 41.5 & 146.0 & 95.1 \\
\hline
\end{tabular}


PEG@CB sample, and the sample temperature rose rapidly. From Figs. 10(b-d), the specimen color quickly turns red in the infrared thermography images. At about $78 \mathrm{~s}$, the sample temperature reaches $55^{\circ} \mathrm{C}$ (initial phase transition temperature). From $0 \mathrm{~s}$ to $78 \mathrm{~s}$, the sensible heat $\left(Q_{s_{1}}\right)$ is stored. From 78 to 99 s, the PEG@CB specimen is in the melting phase transition process and the latent heat $\left(Q_{l}\right)$ is stored by PEG@CB. At 99 s, the melting phase transition process ends. In the case of continued power-on (from 99 to $113 \mathrm{~s})$, the sensible heat $\left(Q_{s_{2}}\right)$ is continuously stored by PEG@CB. Theoretically, according to energy conversion law, the heat generated by Joule's law (Q) is equivalent to the sum of the stored heat $\left(Q_{l}+Q_{s_{1}}+Q_{s_{2}}\right)$ in the PEG@CB and the dissipated energy $\left(Q_{d_{1}}\right)$ during the energy storage process:
$\mathrm{Q}=Q_{l}+Q_{s_{1}}+Q_{s_{2}}+Q_{d_{1}}=m \int_{T=25}^{T=85} C_{P} d T+m \Delta H_{m}+Q_{d_{1}}$

where $m$ is sample weight, $T$ is sample temperature, $C_{p}$ is the heat capacity of sample, and $Q_{d_{1}}$ is the dissipated energy throughout the energy storage process via PCMs. At $113 \mathrm{~s}$, the powder is turned off, the sample temperature drops with time, and the sample color turns from red to blue in the infrared thermography images (as shown in Figs. 10(e-j). From 113 to $275 \mathrm{~s}$, the sample temperature drops from 85 to $50{ }^{\circ} \mathrm{C}$ rapidly and releases the stored sensible heat $\left(Q_{s 1}^{\prime}\right)$. At 275 s, the PEG segments in PEG@CB begin to change from liquid to solid, and begin to release the stored latent heat $\left(\mathrm{Q}_{l}^{\prime}\right)$. At $1646 \mathrm{~s}$, the freezing phase transition process ends, and the sample temperature drops to $35^{\circ} \mathrm{C}$. From 1646 to $2159 \mathrm{~s}$, the stored sensible heat $\left(Q_{s 2}^{\prime}\right)$ is released until the specimen temperature approaches the ambient

Table 3 Thermal parameter of PEG@CB from DSC curves.

\begin{tabular}{ccccc}
\hline & $\Delta H_{m}(\mathrm{~J} / \mathrm{g})$ & $\eta_{m}(\%)$ & $\Delta H_{f}(\mathrm{~J} / \mathrm{g})$ & $\eta_{f}(\%)$ \\
\hline Before & 147.7 & - & 146.0 & - \\
After & 144.5 & 2.2 & 146.6 & -0.4 \\
\hline
\end{tabular}

Table 4 Thermal weight loss parameters for PEG@CB and pure PEG.

\begin{tabular}{cccccc}
\hline & $\mathrm{T}_{5}$ & $T_{\max }$ & \multicolumn{3}{c}{ Weight loss $(\mathrm{wt} \%)$} \\
\cline { 4 - 6 } Specimen & $\left({ }^{\circ} \mathrm{C}\right)$ & $\left({ }^{\circ} \mathrm{C}\right)$ & at $100{ }^{\circ} \mathrm{C}$ & at $250{ }^{\circ} \mathrm{C}$ & at $500{ }^{\circ} \mathrm{C}$ \\
\hline Pure PEG & 380.7 & 421.6 & 0.1 & 0.3 & 98.5 \\
PEG@CB & 382.5 & 413.5 & 0.2 & 0.3 & 87.2 \\
\hline
\end{tabular}

(a)
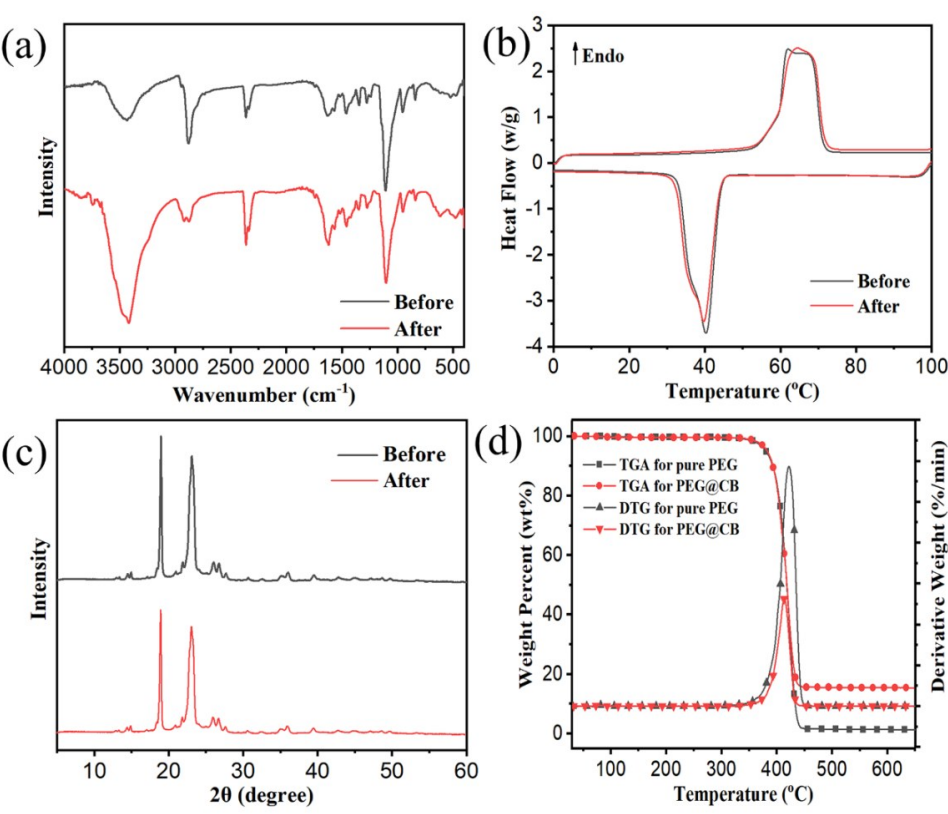

Fig. 6 Curves of PEG@CB before and after 100 thermal cycles, (a) FITR spectra, (b) DSC, (c) XRD, and (d) TG. 
temperature. Similarly, according to energy conservation law, the heat stored by PEG@CB $\left(Q_{l}+Q_{s_{1}}+Q_{s_{2}}\right)$ is equivalent to the sum of the released heat $\left(Q_{s 1}^{\prime}+\mathrm{Q}_{l}^{\prime}+Q_{s 2}^{\prime}\right)$ via the PEG@CB and the dissipated energy $\left(Q_{d_{2}}\right)$ during the energy release process:

$$
\begin{gathered}
Q_{l}+Q_{s_{1}}+Q_{s_{2}}+Q_{d_{1}}=Q_{s 1}^{\prime}+\mathrm{Q}_{l}^{\prime}+Q_{s 2}^{\prime}+Q_{d_{2}}= \\
m \int_{T=85}^{T=25} C_{P} d T+m \Delta H_{f}+Q_{d_{2}}
\end{gathered}
$$

The above results reveal that PEG@CB PCM exhibits excellent electro-to-thermal conversion and thermal energy storage behavior, with which it has a significant potential to store heat thermal energy and convert electro-to-thermal energy.

\section{Conclusion}

In summary, the novel PEG based SSPCC (PEG@CB) was prepared using vacuum impregnation approach. On account of the effects of surface tension and force capillary force, the PEG was retained by as high as $86 \mathrm{wt} \%$ in the porous structure of $\mathrm{CB}$ without leakage. At this percentage, the phase change enthalpy and the relative enthalpy efficiency of PEG@CB are up to $147.7 \mathrm{~J} / \mathrm{g}$ and $95.1 \%$, respectively, which indicate that the $\mathrm{CB}$ is a high efficient supporting material for PEG to prepare PEG based SSPCCs. In addition, PEG@CB shows good thermal properties after 100 thermal cycling, it indicates that the PEG@CB owns good thermal reliability and reusability. Also, PEG@CB shows good thermal stability. Moreover, the volume electrical conductivity of compacted PEG@CB is $0.2 \mathrm{~S} / \mathrm{m}$, it provides excellent electro-to-thermal conversion and thermal energy storage performance for PEG@CB. Taken (a)
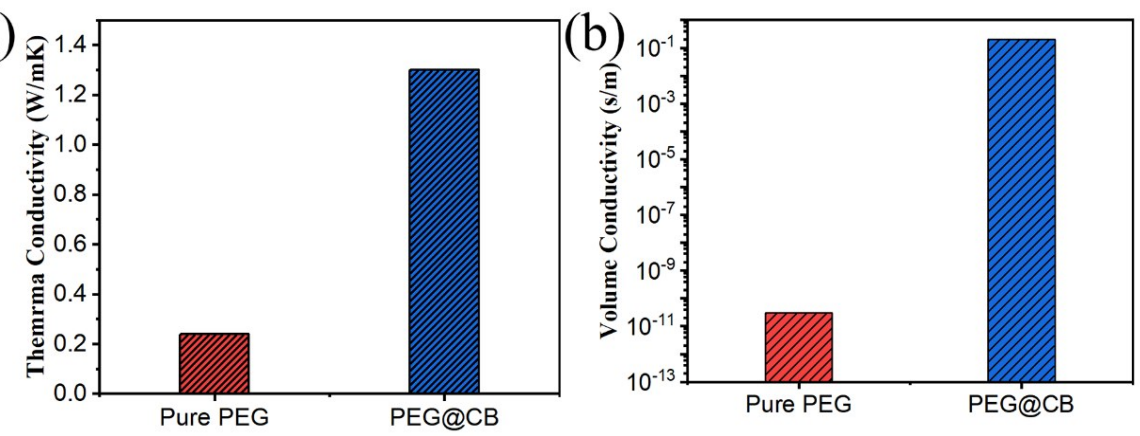

Fig. 7 Thermal conductivity and electrical conductivity of PEG and PEG@CB.

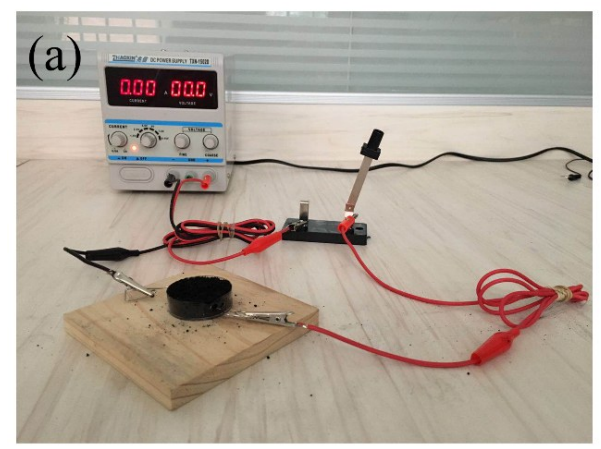

(b)

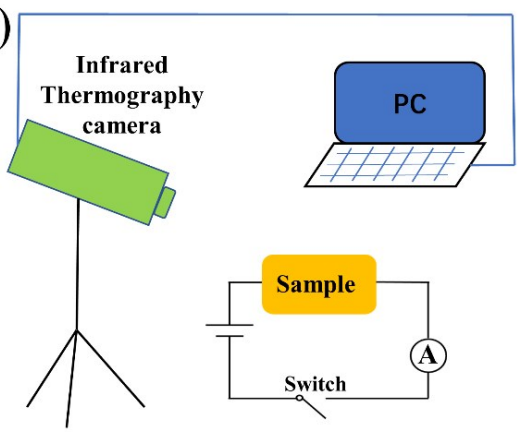

Fig. 8 (a) Digital photo of self-built electro-to-thermal conversion experimental device, (b) the simulation diagram for electro-to-thermal conversion and storage.

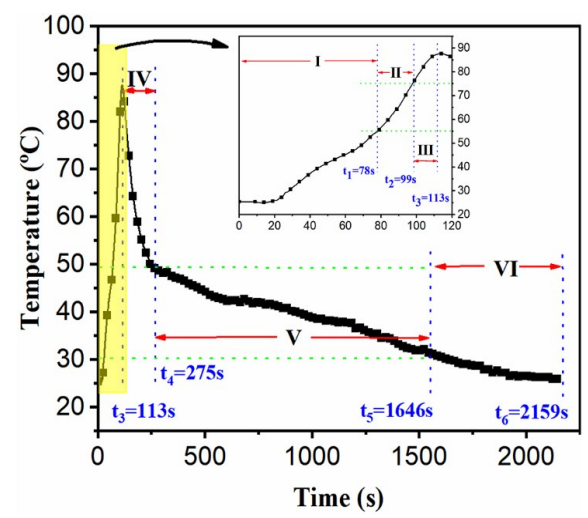

Fig. 9 Temperature curves as a function of time for PEG@CB under electro-tothermalconversion and storage. 

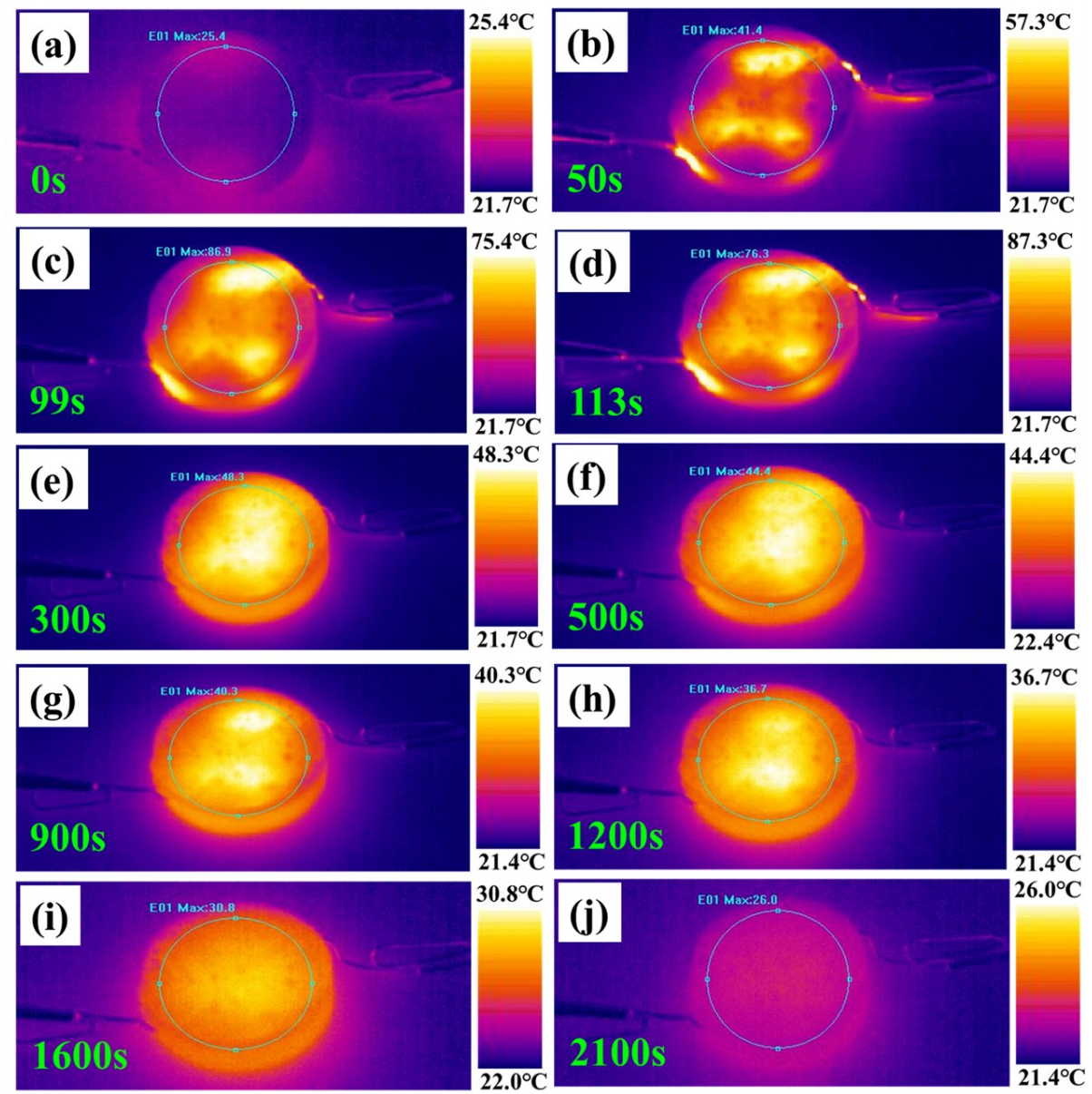

Fig. 10 Infrared thermography images for the electro-to-thermal performance.

together, all the results provide a rich theoretical support for further applications of PEG@CB in the field of peak load regulating of electric power system and corresponding thermal energy storage.

\section{Conflict of Interest}

There are no conflicts to declare.

\section{Acknowledgements}

We acknowledge the National Natural Science Foundation of China (Grant No. 51903092) and the China Postdoctoral Science Foundation funded project (No. 2019M652884), the National Key Research and Development Program of China (GrantNo. 2016YFB0302300), the Natural Science Foundation of Guangdong Province (2016A030313486 and 2018A030313275), the Program of Nanhai Talented Team (201609180006) and the Program of Foshan Innovative Entrepreneurial Team (2016IT100152).

\section{References}

1. Y. Zhao, X. Min, Z. Huang, Y. G. Liu and X. Wu, Energ. Buildings, 2018, 158, 1049-1062.

2. K. Yuan, Y. Zhou, W. Sun, X. Fang and Z. Zhang, Compos. Sci. Technol., 2018, 156, 78-86.

3. J. Yang, L. S. Tang, L. Bai, R. Y. Bao, Z. Liu, B. H. Xie, M. B. Yang and
W. Yang, ACS Sustain. Chem. Eng., 2018, 6, 6761-6770.

4. W. Lin, H. Ren, Z. Ma and L. Yang, J. Clean. Prod., 2019, 233, 1259 1268.

5. Z. Weng, K. Wu, F. Luo, F. Xiao, Q. Zhang, S. Wang and M. Lu, Compos. Part A-Appl. S., 2018, 110, 106-112.

6. H. Abdelrazeq, P. Sobolciak, M. Al-Ali Al-Maadeed, M. Ouederni and I. Krupa, Molecules, 2019, 24, 1217.

7. M. M. S. Al-Azawii, C. Theade, P. Bueno and R. Anderson, Appl. Energ., 2019, 249, 409-422.

8. D. Cabaleiro, F. Agresti, S. Barison, M. A.Marcos, J. I. Prado, S. Rossi, S. Bobbo and L. Fedele, Appl. Therm. Eng., 2019, 159, 113868.

9. J. Yang, L. S. Tang, L. Bai, R. Y. Bao, Z. Y. Liu, B. H. Xie, M. B. Yang and W. Yang, Mater. Horiz., 2019, 6, 250-273.

10. J. Nan, X. Guo, J. Xiao, X. Li, W. Chen, W. Wu, H. Liu, Y. Wang, M. Wu, G. Wang, Small, 2019, 1902085.

11. Z. Pan and X. Ji, J. Power Sources, 2019, 439, 227068.

12. N. Ahmed, K. E. Elfeky, L. Lu and Q. W. Wang, Energ. Convers. Manage., 2019, 189, 14-23.

13. M. E. Arce, M. A. Alvarez Feijoo, A. Suarez Garcia and C. C. Luhrs, Materials, 2018, 11, 195.

14. C. Bott, I. Dressel and P. Bayer, Renew. Sust. Energ. Rev., 2019, 113, 109241.

15. S. Tan, A. P. C. Chan and P. Li, Ind. Eng. Chem. Res., 2019, DOI: 10.1021/acs.iecr.9b02507.

16. R. Cao, S. Chen, Y. Wang, N. Han, H. Liu and X. Zhang, Carbon, 2019, 149, 263-272.

17. X. D. Qi, Y. W. Shao, H. Y. Wu, J. H. Yang and Y. Wang, Compos. Sci. Technol., 2019, 181, 107714.

18. H. Yan, Z. Zhao, W. Ge, N. Zhang and Q. Jin, Ind. Eng. Chem. Res., 
2017, 56, 8408-8415

19. S. Sundararajan, A. B. Samui and P. S. Kulkarni, Ind. Eng. Chem. Res., 2017, 56, 14401-14409.

20. J. Yang, P. Yu, L. S. Tang, R. Y. Bao, Z. Y. Liu, M. B.Yang and W. Yang, Nanoscale, 2017, 9, 17704-17709.

21. L. Jiang, Z. M. Liu, Y. Yuan, Y. J. Wang, J. X. Lei and C. L.Zhou, Energ. Buildings, 2018, 171, 88-99.

22. F. Liu, J. Zhu, J. Liu, B. Ma, W. Zhou and R.Li, Energ. Buildings, 2018, 158, 1781-1789.

23. M. Mu, P. A. M. Basheer, W. Sha, Y. Bai and T. McNally, Appl. Energy, 2016, 162, 68-82.

24. S. Sundararajan, A. B. Samui and P. S. Kulkarni, Thermochim. Acta, 2017, 650, 114-122.

25. X.Guo, Y. Huang and J. Cao, Energ. Buildings, 2018, 158, 1257-1265.

26. X. Jiang, R. Luo, F. Peng, Y. Fang, T. Akiyama and S. Wang, Appl. Energy, 2015, 137, 731-737.

27. L. S. Tang, J. Yang, R. Y. Bao, Z. Y. Liu, B. H. Xie, M. B. Yang and W. Yang, Energ. Convers. Manage., 2017, 146, 253-264.

28. M. Yuan, Y. Ren, C. Xu, F. Ye and X. Du, Renew. Energ., 2019, 136, 211-222.

29. Y. Wang, A. Du Pasquier, D. Li, P. Atanassova, S. Sawrey and M. Oljaca, Carbon, 2018, 133, 1-5.

30. J. Macutkevic, P. Kuzhir, A. Paddubskaya, S. Maksimenko, J. Banys, A. Celzard, V. Fierro, E. Stefanutti, A. Cataldo, F. Micciulla and S. Bellucci, J. Nanosci. Nanotechno., 2013, 13, 5434-5439.

31. G. Qi, J. Yang, R. Bao, D. Xia, M. Cao, W. Yang, M. Yang and D. Wei,
Nano Res., 2016, 10, 802-813.

32. Y. Deng, J. Li, T. Qian, W. Guan, Y. Li and X. Yin, Chem. Eng. J., 2016, 295, 427-435.

33. T. Qian, J. Li, H. Ma and J. Yang, Polym. Composite., 2016, 37, 854860 .

34. T. Qian, J. Li and Y. Deng, Sci. Rep-UK, 2016, 6, 32392.

35. X. Zhang, R. Wen, C. Tang, B. Wu, Z. Huang, X. Min, Y. Huang, Y. Liu, M. Fang and X. Wu, Energ. Buildings, 2016, 130, 113-121.

36. L. Feng, J. Zheng, H. Yang, Y. Guo, W. Li and X. Li, Sol. Energ. Mater. Sol. C., 2011, 95, 644-650.

37. D. Zhang, M. Chen, Q. Liu, J. Wan and J. Hu, Materials, 2018, 11, 818.

38. C. Wang, Z. Cai, K. Chen, J. Huang, T. Wang, Energ Storage, 2019, 1, 45.

39. Y. Zhou, D. Sheng, X. Liu, C. Lin, F. Ji, L.Dong, S. Xu and Y. Yang, Sol. Energ. Mater. Sol. C., 2018, 174, 84-93.

40. M. Alfe, V. Gargiulo, R. Di Capua, F. Chiarella, J. N. Rouzaud, A. Vergara and A. Ciajolo, ACS Appl. Mater. Interfaces, 2012, 4, 4491-8.

41. X. Lu, C. Fang, X. Sheng, L. Zhang and J. Qu, Ind. Eng. Chem. Res., 2019, 58, 3024-3032.

42. F. Xue, Y. Lu, X. D. Qi, J. H. Yang and Y. Wang, Chem. Eng. J., 2019, 365, 20-29.

Publisher's Note Engineered Science Publisher remains neutral with regard to jurisdictional claims in published maps and institutional affiliations. 